\title{
Selection Criteria for Determination of Optimal Reconstruction Method for Cu-64 Trastuzumab Dosimetry on Siemens Inveon PET Scanner
}

\author{
Seonhwa Lee ${ }^{1,2} \oplus$, Hyeongi Kim ${ }^{1} \oplus$, Ye-rin Kang ${ }^{3,4}$, Hyungwoo Kim ${ }^{3,5}$, Jung Young Kim ${ }^{1}$, \\ Yong-Jin Lee ${ }^{1}$, Jung Min Kim ${ }^{2}$ and Jin Su Kim ${ }^{1,3, *(1)}$ \\ 1 Division of RI Application, Korea Institute of Radiological and Medical Sciences (KIRAMS), 75 Nowon-ro, \\ Nowon-gu, Seoul 01812, Korea; 2015010289@korea.ac.kr (S.L.); hyeongi@kirams.re.kr (H.K.); \\ jykim@kirams.re.kr (J.Y.K.); yjlee@kirams.re.kr (Y.-J.L.) \\ 2 Department of Bio-Convergence Engineering, Korea University, Seoul 02856, Korea; minbogun@korea.ac.kr \\ 3 Radiologicial and Medico-Oncological Sciences Major, University of Science and Technology (UST), \\ Seoul 01812, Korea; kucs25@korea.ac.kr (Y.-r.K.); hungwoo.kim@khu.ac.kr (H.K.) \\ 4 School of Health and Environmental Science, College of Health Science, Korea University, Seoul 02856, Korea \\ 5 College of Korean Medicine, Kyung Hee University, Seoul 02454, Korea \\ * Correspondence: kjs@kirams.re.kr; Tel.: +82-2-970-1661
}

Received: 28 February 2019; Accepted: 10 April 2019; Published: 14 April 2019

\begin{abstract}
The goal of this study was to suggest criteria for the determination of the optimal image reconstruction algorithm for image-based dosimetry of Cu-64 trastuzumab PET in a mouse model. Image qualities, such as recovery coefficient (RC), spill-over ratio (SOR), and non-uniformity (NU), were measured according to National Electrical Manufacturers Association (NEMA) NU4-2008. Mice bearing a subcutaneous tumor $\left(200 \mathrm{~mm}^{3}\right.$, HER2 NCI N87) were injected with monoclonal antibodies (trastuzumab) with $\mathrm{Cu}-64$. Preclinical mouse PET images were acquired at 4 time points after injection $(2,15,40$ and $64 \mathrm{~h})$. Phantom and Cu-64 trastuzumab PET images were reconstructed using various reconstruction algorithms (filtered back projection (FBP), 3D reprojection algorithm (FBP-3DRP), 2D ordered subset expectation maximization (OSEM 2D), and OSEM 3D maximum a posteriori (OSEM3D-MAP)) and filters. The absorbed dose for the tumor and the effective dose for organs for $\mathrm{Cu}-64$ trastuzumab PET were calculated using the OLINDA/EXM program with various reconstruction algorithms. Absorbed dose for the tumor ranged from $923 \mathrm{mGy} / \mathrm{MBq}$ to $1830 \mathrm{mGy} / \mathrm{MBq}$ with application of reconstruction algorithms and filters. When OSEM2D was used, the effective osteogenic dose increased from 0.0031 to 0.0245 with an increase in the iteration number ( 1 to 10). In the region of kidney, the effective dose increased from 0.1870 to 1.4100 when OSEM2D was used with iteration number 1 to 10 . To determine the optimal reconstruction algorithms and filters, a correlation between RC and NU was plotted and selection criteria $(0.9<\mathrm{RC}<1.0$ and $<10 \%$ of NU) were suggested. According to the selection criteria, OSEM2D (iteration 1) was chosen for the optimal reconstruction algorithm. OSEM2D (iteration 10) provided $154.7 \%$ overestimated effective dose and FBP with a Butterworth filter provided $20.9 \%$ underestimated effective dose. We suggested OSEM2D (iteration 1) for the calculation of the effective dose of $\mathrm{Cu}-64$ trastuzumab on an Inveon PET scanner.
\end{abstract}

Keywords: Cu-64; trastuzumab; dosimetry; NEMA NU4-2008 guideline; Inveon PET

\section{Introduction}

Immuno-PET uses monoclonal antibodies (mAbs) that are labeled with positron-emitting radionuclides. Immuno-PET can be applied for tailored patient management, dose calculation 
in radioimmunotherapy (RIT), and development of antibody-based drugs [1-3]. Cu-64 decays by both $\beta+(655 \mathrm{keV}, 17.4 \%)$ and $\beta-(573 \mathrm{keV}, 39.0 \%)$ emission, making it suitable for both immuno-PET and radioimmuno-therapy (RIT) [4]. One of the widely used clinical trials was $\mathrm{Cu}-64$ trastuzumab [5-7]. The safety of $\mathrm{Cu}-64$ trastuzumab in HER2-positive breast cancer has been investigated using radiation dosimetry [8].

Image-based radiation dosimetry in nuclear medicine provides information on the absorption of energy from ionizing radiation and on the effect of radiation damage to living tissues. Image-based dosimetry could be used to assess the safety and efficacy of immuno-PET [9-12]. For the implementation of image-based dosimetry, the cumulative activity ( $\tilde{\mathrm{A}})$ should be calculated. The $\tilde{\mathrm{A}}$ is the product of the amount of activity present in the source organ and the length of time during which this activity is present $[11,12]$. To calculate the amount of cumulative activity, the region of interest (ROI) was drawn on the reconstructed PET or SPECT data. The dimensions of the matrix, the zoom factors, the spatial filters, and the reconstruction algorithms are factors that determine the spatial resolution of PET [13].

Performance measurement studies using the National Electrical Manufacturers Association (NEMA) standard NU 4-2008 [14] for a preclinical scanner provided us with information on spatial resolution, sensitivity, and image quality, such as non-uniformity and recovery coefficients [14-17]. In addition, a comparative study regarding reconstruction algorithms with filters was performed in terms of spatial resolution, recovery coefficient, and spill-over ratio, according to the NEMA standard NU4 [18-20]. Comparative studies for quantification of Zr-89 and I-124 were also performed [18-21]. However, there are no results from the investigation of reconstruction algorithms when various reconstruction algorithms were applied to the dosimetry study.

In this study, we investigated the effect of reconstruction algorithms on the result of image-based dosimetry using $\mathrm{Cu}-64$ trastuzumab PET on a Siemens Inveon PET scanner. In addition, we suggested criteria for the determination of the optimal reconstruction algorithms. To our best knowledge, our investigation is the first paper on selection criteria for determination of the optimal reconstruction algorithm and the relationship between the reconstruction algorithm and image-based radiation dosimetry on immuno-PET small animal studies.

\section{Experimental Section}

\subsection{Ethical Statement}

This article does not contain any studies involving human participants. The study was approved in accordance with institutional IACUC protocols.

\subsection{PET Scanner}

All PET scans in this study were performed on a Siemens Inveon PET scanner (Siemens Medical Solutions, Tarrytown, NY, USA) [16].

\subsection{Reconstruction Algorithms}

All reconstruction algorithms implemented on the Inveon PET scanner, such as filtered back projection (FBP), 3D reprojection algorithm (FBP-3DRP), 2D ordered subset expectation maximization (OSEM 2D), and OSEM 3D maximum a posteriori (OSEM3D-MAP), were used for phantom and preclinical studies.

For the FBP reconstruction, the ramp, Butterworth, Hamming, Hanning, Parzen, and Shepp-Logan filters were used. The cutoff frequency was 0.5. For OSEM2D algorithm, the subset number was 16 and the iteration numbers from 1 to 10 were used. OSEM3D is one of the most widely used reconstruction methods. When the iteration number was set to 0 during OSEM3D-MAP reconstruction, OSEM3D without MAP reconstruction method could be implemented on Inveon PET scanner. For the OSEM3D-MAP reconstruction, various smoothing factors $(\beta)$ were used with MAP iteration number 18. MAP Iteration 18 was used on the basis of the result of Lasnon and colleagues. According to 
the results of Lasnon and colleagues (Figure 1 from ref. [18]), there was no significant change in the non-uniformity, recovery coefficient (RC), or spill-over ratio (SOR) with iteration number for the OSEM3D-MAP reconstruction. Both MAP and this implementation of OSEM3D contained information such as the scanner geometry and point spread function (PSF) of the detector response for the various angles that the $511 \mathrm{keV}$ photons struck the detector. This information was not contained in any of the other reconstruction algorithms (FBP, 3DRP, and OSEM2D). The scanner geometry information was stored in the Pmatrix files, whereas the PSF information was stored in the blur kernels. MAP blur kernels were two-dimensional, whereas the OSEM3D blur kernels was one-dimensional, which led to better resolution recovery for MAP than for OSEM3D. In addition, MAP used a smoothing parameter ( $\beta$ value) that constrained neighboring voxels in the reconstructed image.

Siemens also provided "MAP iteration 18" as a default option instead of OSEM3D during OSEM3D-MAP reconstruction algorithm on Inveon PET scanner. Therefore, in this present study, we focused on the comparison of $\beta$ smoothing factor during OSEM3D-MAP reconstruction.

To measure the effect of post-smoothing with Gaussian kernels, various Gaussian smoothing kernels $(1.5 \mathrm{~mm}, 3.0 \mathrm{~mm}, 4.5 \mathrm{~mm}$, and $6.0 \mathrm{~mm})$ were applied to reconstructed PET data. OSEM2D with iteration 1 and OSEM3D-MAP with $\beta=1.5$ were representatively selected. The results of the effect of Gaussian smoothing were described in Figure A2 and Appendix A. We used Inveon reconstruction software suite (IAW version 1.4.3) for reconstruction.

\subsection{Attenuation and Scatter Corrections}

We assessed the effects of attenuation correction (AC), and AC and scatter correction (SC) in PET. All emission data were acquired within an energy window of 350-650 keV. Transmission (TX) data were acquired using a Co-57 source (activity, $136 \mathrm{MBq}$ ) for $15 \mathrm{~min}$. TX PET data were acquired within an energy window of 120-125 keV. Attenuation coefficients were scaled to $511 \mathrm{keV}$ automatically during histogramming. Blank data were also acquired using a Co-57 source for $3 \mathrm{~h}$. Normalization data were acquired for $10 \mathrm{~h}$. All TX and blank data were histogrammed by single slice rebinning. All emission data and normalization data were sorted into a 3D sinogram. SC was performed for all datasets using a single scatter simulation (SSS) scatter correction algorithm. SC was implemented using Inveon reconstruction software suite (IAW version 1.4.3).

\subsection{Phantom Studies}

To determine the optimal reconstruction parameters for Cu-64 trastuzumab PET, phantom studies were performed according to the NEMA NU4 guidelines [14]. The recovery coefficient (RC), non-uniformity (NU), and spill-over ratio (SOR) were measured. Image quality phantom data for $\mathrm{Cu}-64$ was acquired for $20 \mathrm{~min}$ within an energy window of $\sim 350-650 \mathrm{keV}$. Radioactivity was $3.7 \mathrm{MBq}$. Normalization, AC, and SC were applied. The matrix size was $128 \times 128 \times 159$ and the voxel size was $0.776 \times 0.776 \times 0.796 \mathrm{~mm}^{3}$. NU, RC, and SOR were calculated for data that were only corrected for normalization, as well as for data that were corrected for AC and AC and SC effects. In this study, the term "partially corrected" meant that normalization, dead-time, and random correction were performed.

\subsubsection{Non-Uniformity}

To determine the NU, a cylindrical volume of interest (VOI) (length $10 \mathrm{~mm}$ and diameter $22.5 \mathrm{~mm}$ ) was drawn at the center of a uniform region. Mean value and standard deviation (SD) were measured in VOI. Non-uniformity was expressed in percent SD (\% SD: Percent of standard deviation divided by mean).

\subsubsection{Recovery Coefficient}

The RC is a measure of how well a PET scanner system can recover the true radioactivity concentration in the corresponding uniform area. RC can be obtained as following: 
$\mathrm{RC}=$ measured peak activity concentration/true activity concentration.

To determine the $\mathrm{RCs}$, five fillable rods of a radioactive source $(1,2,3,4$, and $5 \mathrm{~mm}$ in diameter) were used at the bottom of the cylinder (length: $20 \mathrm{~mm}$, diameter: $30 \mathrm{~mm}$ ). Circular ROIs were drawn around each rod. The size of the ROI was twice the physical diameter of the rods.

\subsubsection{Spill-Over Ratio}

The SOR was defined as the ratio of the mean value in each cold cylinder to the mean value in the uniform area. The upper part of the uniform region was a cold region consisting of two empty cylinders (length: $15 \mathrm{~mm}$, inner diameter: $8 \mathrm{~mm}$, outer diameter: $10 \mathrm{~mm}$ ). One space was filled with air and the other space with nonradioactive water. Although both cylinders were nonradioactive, scattered coincidence and random coincidence contributed to activity in the nonradioactive cylinder. In these non-radioactive regions, SOR values could be calculated to determine the effect of scattered or random coincidences in the nonradioactive region. To calculate the SOR, 2 cylindrical VOIs (length: $7.5 \mathrm{~mm}$, diameter: $4 \mathrm{~mm}$ ) were drawn in the air- and water-filled compartments.

\subsection{Quantification of Cu-64 Trastuzumab PET}

\subsubsection{Cell Culture and Tumor Xenograft in Mice}

NCI-N87 HER2+ cancer cell lines, obtained from the American Type Culture Collection (ATCC), were maintained in Roswell Park Memorial Institute (RPMI) medium containing 10\% fetal bovine serum (FBS) with antibiotics (Sigma, St. Louis, MO, USA) at $37^{\circ} \mathrm{C}$ in a humidified $5 \% \mathrm{CO}_{2}$ incubator. The NCI-N87 cells $(5 \times 106)$ were injected subcutaneously into the right flank of female athymic BALB/C nude mice $(n=5)$ aged 5 weeks (Shizuoka Laboratory Animal Center, Hamamatsu, Japan). Tumor size was measured using a digital caliper and tumor volume was calculated by applying the formula width ${ }^{2}$ $\times$ length $\times 0.4$. All experiments were performed in accordance with the institutional guidelines of the Korea Institute of Radiological and Medical Sciences (KIRAMS).

\subsubsection{Radiolabeling of $\mathrm{Cu}-64-\mathrm{DOTA}-$ Trastuzumab}

Trastuzumab (20 mg) was added to DOTA-NHS-ester ( $1 \mathrm{mg}$, Macrocyclics) in $800 \mu \mathrm{L}$ of 1 M sodium bicarbonate buffer ( $\mathrm{pH} 8.5$ ) and mixed gently for $24 \mathrm{~h}$ at $4{ }^{\circ} \mathrm{C}$. Unconjugated chelator was removed using PD-10 column with $1 \mathrm{mM}$ sodium acetate ( $\mathrm{pH}$ 5.5). Cu-64 was produced at KIRAMS by 50-MeV cyclotron irradiation. Trastuzumab (Herceptin; F. Hoffmann-La Roche, Basel, Switzerland) was labeled with $\mathrm{Cu}-64$ by conjugation with DOTA. ${ }^{64} \mathrm{CuCl}_{2}(370 \mathrm{MBq})$ was added to $1 \mathrm{mg}$ of DOTA-trastuzumab in $1 \mathrm{mM}$ sodium acetate ( $\mathrm{pH}$ 5.5) and incubated for $1 \mathrm{~h}$ at $37^{\circ} \mathrm{C}$. Instant thin-layer chromatography on silica gel (solvent—citric acid) showed that the radiolabeling yield was $\geq 95 \%$.

\subsubsection{Cu-64 Trastuzumab PET}

Three to four weeks after the implantation of the tumor, the tumor size reached about $200 \mathrm{~mm}^{3}$; $\mathrm{Cu}-64$ DOTA trastuzumab $(400 \mu \mathrm{Ci} / 150 \mu \mathrm{g})$ was injected intravenously through a tail vein. Mice were anesthetized with $2 \%$ isoflurane in $100 \%$ oxygen (Forane solution; ChoongWae Pharma, Seoul, Korea). PET data were acquired during $15 \mathrm{~min}$ at time points of $2 \mathrm{~h}, 15 \mathrm{~h}, 40 \mathrm{~h}$, and $64 \mathrm{~h}$ after injection of $\mathrm{Cu}-64$ trastuzumab. PET data were reconstructed using various algorithms and filters to compare their performance.

For the acquisition of anatomical image, $\mathrm{X}$-ray CT for mice was acquired with full rotation and 180 projections using the Inveon system. Exposure time was $200 \mathrm{~ms}$, and the estimated scan time was for $504 \mathrm{~s}$ X-ray CT. X-ray CT data were reconstructed using Feldkamp reconstruction with Shepp-Logan filtering. Effective pixel size of the reconstructed X-ray CT image was $109.69 \mu \mathrm{m} \times$ $109.69 \mu \mathrm{m}$. X-ray CT was used for the delineation of ROI. ROIs were delineated in the regions of the brain, lungs, liver, stomach, intestines, kidney, and tumor. The size of the ROIs ranged from 0.0067 to $0.066 \mathrm{~cm}^{2}$. After delineation of ROIs on X-ray CT, ROIs were copied to Cu-64 trastuzumab PET data. 
The maximum value from the ROI region was extracted and the percentage of injected dose/gram (\% $\mathrm{ID} / \mathrm{g}$ ) was calculated.

\subsubsection{Dosimetry}

The radiation dose per unit of administered activity $(\mathrm{mSv} / \mathrm{MBq})$, the effective dose in organ, and the absorbed dose for the tumor region on $\mathrm{Cu}-64$ trastuzumab PET in mice were calculated using OLINDA/EXM software (OLINDA; Vanderbilt University, Nashville, TN, USA) [9]. Time activity curves (TACs) were obtained for each organ. Decay-uncorrected TACs were derived and cumulative activity was obtained from the area under the curve (AUC) for TACs. For each source organ, the residence time was calculated by dividing the cumulative activity by the total injected dose. Olinda used models for an average adult male or female human. Scaling of mouse-derived time activity data was applied before entering residence time data into Olinda. The scaling method was described in our previous work [10].

For the calculation of the absorbed dose in the tumor, a sphere model in OLINDA was used. Tumor volume was calculated on $\mathrm{Cu}-64$ trastuzumab PET data with multiple slices of ROIs. Tumor mass was calculated under the assumption of $1 \mathrm{~g} / \mathrm{mL}$. The absorbed S-value for each tumor volume was calculated with scaling by mass. A non-linear fitting between the S-value and the mass was used, because linear interpolation could provide too large a value of $S$.

The effective dose for each organ and the absorbed dose for the tumor region were calculated using various reconstruction algorithms and filters.

\section{Results}

\subsection{Non-Uniformity}

Figure 1 shows the result of NUs, expressed in \% SD, for various reconstruction algorithms and filters. The lowest NU (lowest \% SD) was achieved when FBP with a Parzen filter was used. The values of NU were $5.00 \%$ for partially corrected, $4.95 \%$ for AC corrected, and $5.39 \%$ for AC and SC corrected, respectively. When OSEM2D reconstruction method was used, NUs increased with an increase of the iteration number. This means that images become noisier with increasing number of iterations. For analytical reconstruction methods such as FBP and FBP-3DRP, the differences in NUs between the methods were negligible $(<3 \%)$ after AC was applied compared to partially corrected images. The NUs increased by $13 \%$ when AC and SC were applied. For iterative reconstruction methods, such as OSEM2D or OSEM3D-MAP reconstruction methods, the differences in NUs were within 5\% between partially corrected images and AC images. When AC and SC were applied, the NUs increased by $23 \%$ for the OSEM2D reconstruction method. 


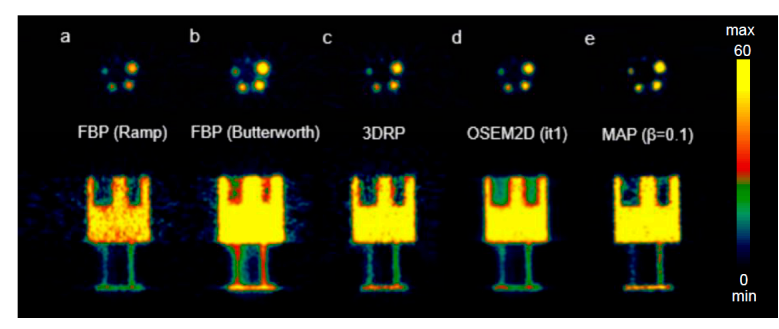

(a)

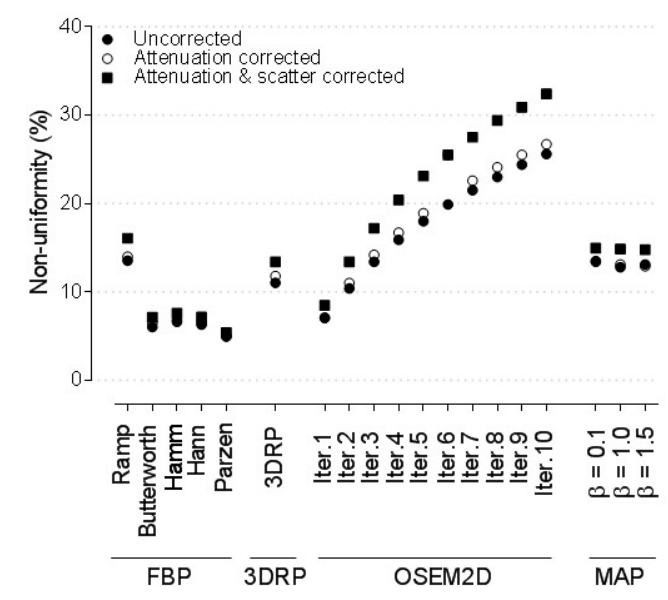

(b)

Figure 1. (a) Representative Cu-64 PET data of National Electrical Manufacturers Association (NEMA) NU4-2008 phantom. The reconstruction algorithms used were filtered back projection (FBP) (ramp filter), FBP (Butterworth filter), FBP-3DRP, 2D ordered subset expectation maximization (OSEM 2D) (iteration 1), and OSEM3D-MAP $(\beta=0.1)$. These data were presented for partially corrected data. (b) Non-uniformity for various reconstruction algorithms, such as FBP, FBP-3DRP, OSEM2D, and OSEM3D-MAP reconstruction with filters. Normalization, dead-time, and random corrections have been applied to all PET raw data. The effects of attenuation correction (AC), and AC and scatter correction (SC) corrections in PET were also provided. The term "partially corrected" meant that normalization, dead-time, and random correction were performed. AC: attenuation correction, SC: scatter correction, 3DRP: FBP-3DRP, MAP: OSEM3D-MAP.

\subsection{Recovery Coefficient and Non-Uniformity}

Figure 2a-c shows the RCs when partially corrected, with AC applied, and AC and SC applied, respectively. The RC analysis for rods with a size of $1 \mathrm{~mm}$ was not performed, because they were not discernible on the PET image. Figure $2 \mathrm{~d}$ shows the RCs of $3 \mathrm{~mm}$ rods for various reconstruction methods when AC and SC were applied. When FBP was used with ramp filters for Cu-64 PET imaging, the highest $R C$ values were $0.50,0.86,1.04$, and 1.17 for $2,3,4$, and $5 \mathrm{~mm}$ rods, respectively. Regarding the iterative reconstruction algorithms for Cu-64 PET imaging, the highest RC values were $0.42,0.88,2.04$, and 2.09 for 2, 3, 4, and $5 \mathrm{~mm}$ rods when OSEM2D (iteration 10) was used. Regarding OSEM3D-MAP reconstruction algorithms, the highest $R C$ values were $0.49,1.29$. 1.46, and 1.21 for $2,3,4$, and $5 \mathrm{~mm}$ rods.

Figure $3 \mathrm{a}-\mathrm{d}$ shows the $\mathrm{RC}$ for $3 \mathrm{~mm}$ rods plotted against the NU when partially corrected, with AC, and AC and SC, respectively. OSEM2D (iteration 1) provided an RC value of 0.92 and an NU of $7.0 \%$ for partially corrected and OSEM2D (iteration 1) provided an RC value of 0.94 and an NU of $7.1 \%$ for AC. However, when AC \& SC corrections were applied, the RC value deteriorated to 0.70 for OSEM2D (iteration 1). 


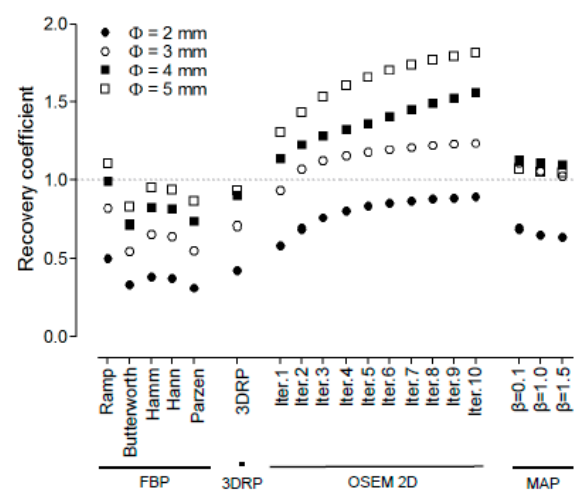

(a)

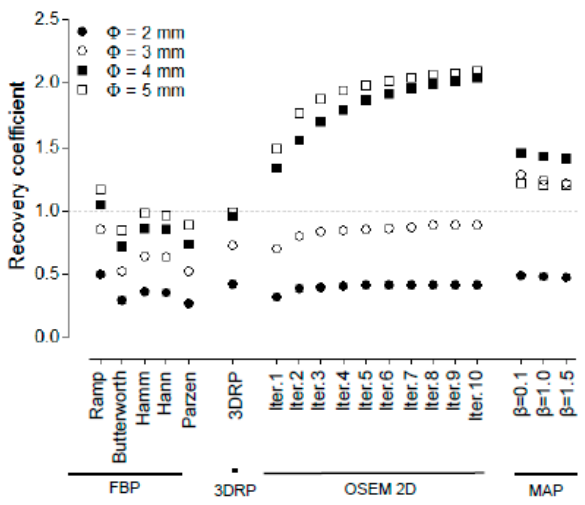

(c)
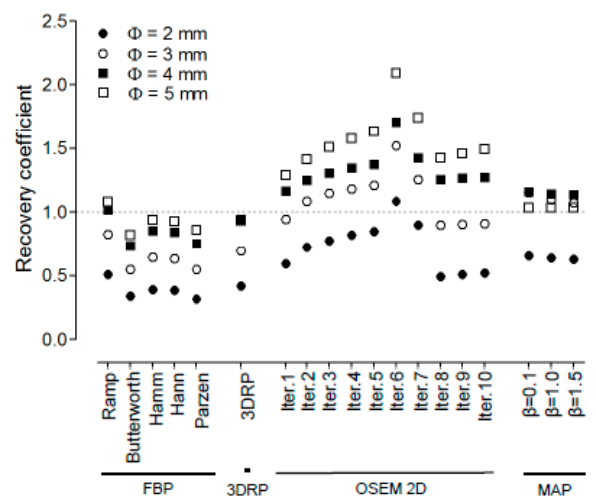

(b)

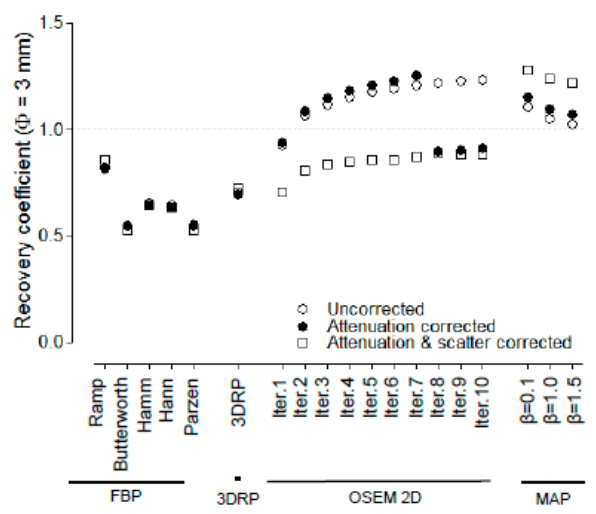

(d)

Figure 2. Recovery coefficient for various reconstruction algorithms, such as FBP, FBP-3DRP, OSEM2D, and OSEM3D-MAP reconstruction with filters. All data were presented for (a) partially corrected data, (b) AC, and (c) AC and SC data. The diameter of the rods were 2, 3, 4, and $5 \mathrm{~mm} .1 \mathrm{~mm}$ diameter of rod was not visible. (d) RCs of $3 \mathrm{~mm}$ rods for various reconstruction methods. All data were presented for partially corrected data, AC and AC \& SC data. 3DRP: FBP-3DRP, MAP: OSEM3D-MAP.

(A)

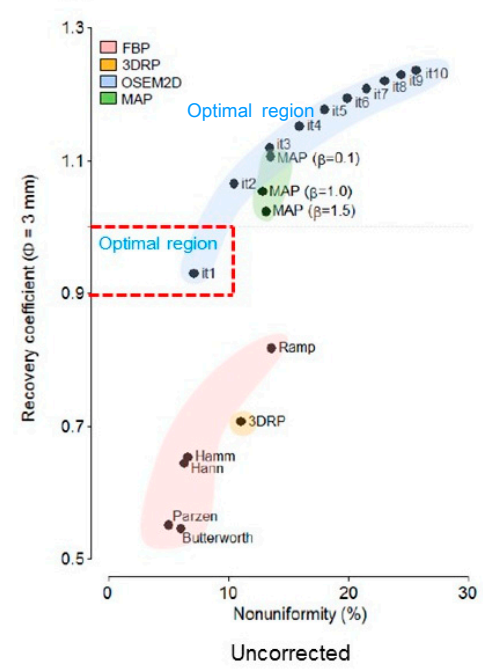

(B)

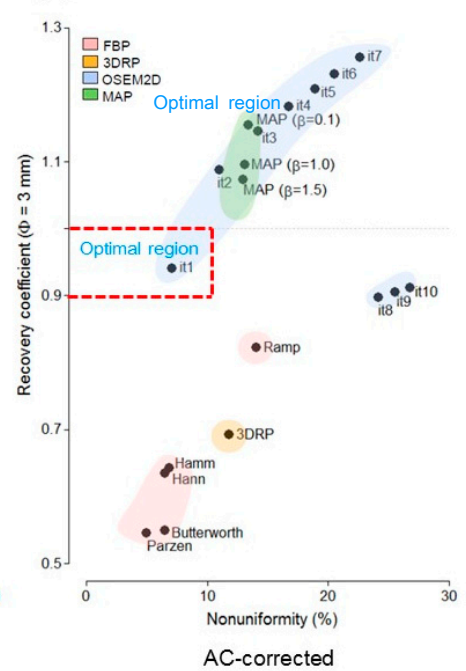

(C)

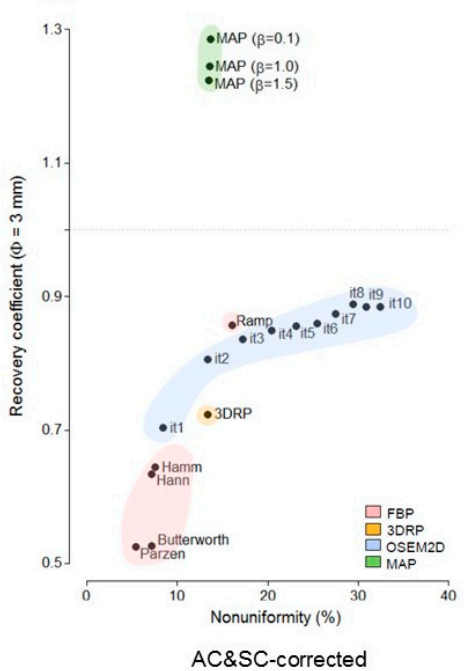

Figure 3. Plot of non-uniformity versus recovery coefficient (rod diameter is $3 \mathrm{~mm}$ ) for various reconstruction algorithms, such as FBP, FBP-3DRP, OSEM2D, and OSEM3D-MAP reconstruction with filters. All data were presented for (a) partially corrected data, (b) AC corrected, and (c) AC and SC corrected data. The optimal criteria $(0.9<\mathrm{RC}<1.0$ and $\mathrm{NU}<10 \%)$ are marked in the red box. 3DRP: FBP-3DRP, MAP: OSEM3D-MAP. 


\subsection{Spill-Over Ratio}

Figure 4 shows the SOR in the air and water compartments. For both the air and water compartments, FBP-3DRP provided the lowest SOR among the reconstruction algorithms for partially corrected, AC, and AC and SC.

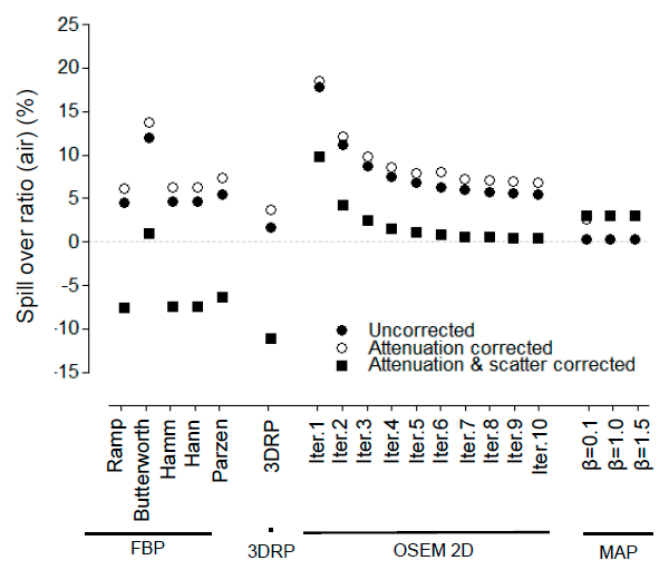

(a)

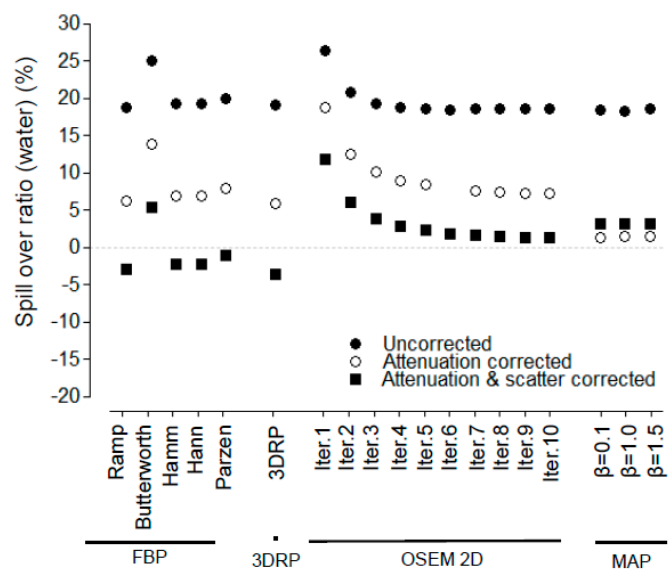

(b)

Figure 4. Spill-over ratio for various reconstruction algorithms, such as FBP, FBP-3DRP, OSEM2D, and OSEM3D-MAP reconstruction with filters. All data were presented for partially corrected data, AC corrected, and AC and SC corrected. Spill-over ratio for (a) air and (b) water. The term "partially corrected" meant that normalization, dead-time, and random correction were performed. 3DRP: FBP-3DRP, MAP: OSEM3D-MAP.

\subsection{Cu-64 Trastuzumab PET}

Figure 5a shows the PET images of $\mathrm{Cu}-64$ trastuzumab at 2, 15, 40, and $64 \mathrm{~h}$ after administration of Cu-64 trastuzumab using a HER2 + NCI N87 mouse model. Figure 5b shows the time activity curve and the corresponding AUC of the tumor region for various reconstruction algorithms. We confirmed there was no presence of negative values in the TAC, which was allowed for FBP and FBP-3DRP algorithms. The smoothing that was imposed by the OSEM3D-MAP algorithms would be the reason for the lower TAC values compared to OSEM2D. Lower values of TAC were obtained when a higher value of smoothing factor $(\beta)$ was applied during MAP reconstruction. 


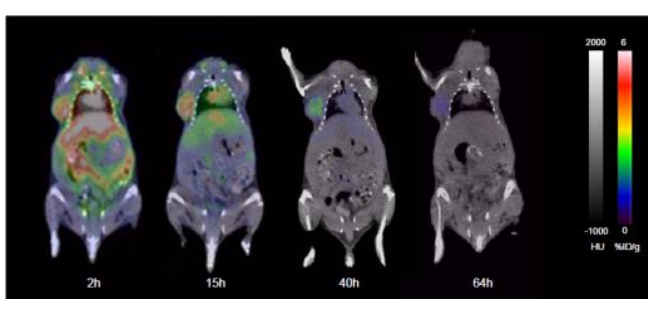

(a)

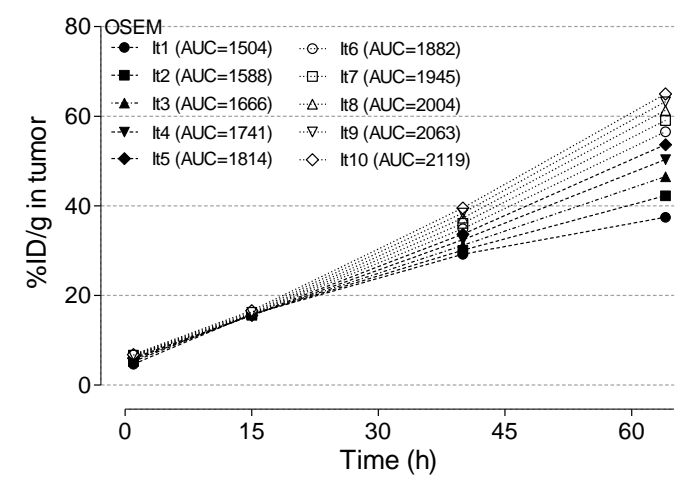

(c)

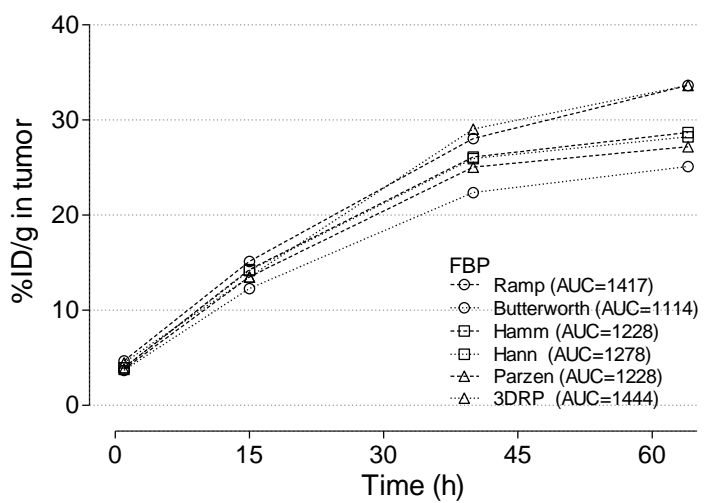

(b)

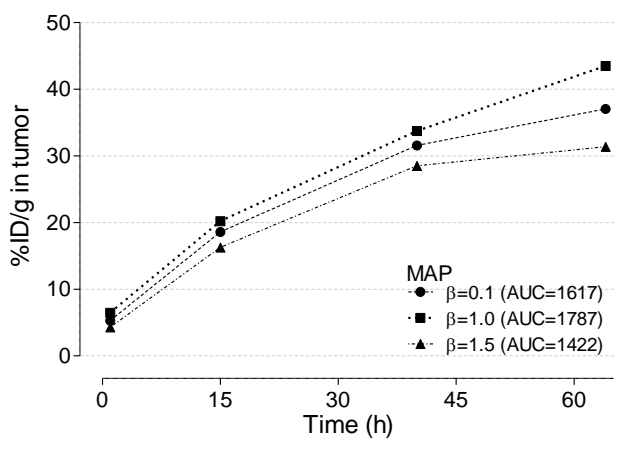

(d)

Figure 5. (a) PET image for 2, 15, 40, and $64 \mathrm{~h}$ after injection of $\mathrm{Cu}-64$ trastuzumab. The PET data was overlaid on the X-ray CT data. \% ID/g versus the time in the tumor region. \% ID/g was calculated using various reconstruction algorithms, such as (b) FBP and FBP-3DRP, (c) OSE2D, and (d) OSEM3D-MAP. 3DRP: FBP-3DRP, MAP: OSEM3D-MAP.

\subsection{Radiation Dosimetry Using Various Reconstruction Algorithms and Filters}

Table 1 shows the results of radiation dosimetry for $\mathrm{Cu}-64$ trastuzumab PET. The absorbed dose for the tumor ranged from $923 \mathrm{mGy} / \mathrm{MBq}$ to $1830 \mathrm{mGy} / \mathrm{MBq}$. The absorbed dose for the tumor was 1200, 923, 1020, and 1020 mGy/MBq when FBP was used with ramp, Butterworth, Hamm, Hann, and Parzen filters, respectively. When OSEM2D was used, the absorbed dose to the tumor increased from 1320 to $1830 \mathrm{mGy} / \mathrm{MBq}$ with increasing number of iterations. Effective doses for organs have the same tendency. The effective dose for osteogenic was 0.0023 and 0.0047 when FBP reconstruction with Butterworth filter or Parzen filter was used, respectively. When OSEM2D was used, the effective dose for osteogenesis increased from 0.0031 to 0.0245 with increasing number of iterations. In the region of the kidney, the effective dose increased from 0.1870 to 1.4100 when OSEM2D was used. 
Table 1. Radiation dose estimates of the tumor and organs from mice injected with $\mathrm{Cu}-64$ DOTA trastuzumab.

\begin{tabular}{|c|c|c|c|c|c|c|c|c|c|c|c|c|c|c|c|c|c|c|c|}
\hline & \multicolumn{5}{|c|}{ FBP } & \multirow[t]{2}{*}{ 3DRP } & \multicolumn{10}{|c|}{ OSEM2D } & \multicolumn{3}{|c|}{ OSEM3D-MAP } \\
\hline & Ramp & Butterworth & Hamm & Hann & Parzen & & Iter.1 & Iter.2 & Iter.3 & Iter.4 & Iter.5 & Iter.6 & Iter.7 & Iter. 8 & Iter.9 & Iter.10 & $\beta=0.1$ & $\beta=1.0$ & $\beta=1.5$ \\
\hline $\begin{array}{l}\text { Tumor (mGy/MBq) } \\
\text { Organ (mSv/MBq) }\end{array}$ & 1200 & 923 & 1020 & 1060 & 1020 & 1250 & 1320 & 1340 & 1440 & 1480 & 1560 & 1620 & 1660 & 1710 & 1760 & 1830 & 1380 & 1500 & 1180 \\
\hline Adrenals & 122 & 0.0094 & 0.0106 & 0.0104 & 0.0175 & 0.0121 & 0122 & 0.0140 & 0.0159 & 0.0175 & 0.0190 & 0.0206 & 0.0219 & 0.0227 & 0.0259 & 0.0911 & 0.0127 & 0.0141 & 0.0113 \\
\hline Brain & 327 & 0.0216 & 0.0222 & 0.0215 & 0556 & 0.0236 & 0.0263 & 0.0367 & 0.0466 & 0.0556 & 0.0642 & 0.0756 & 0.0775 & 0.0823 & 0.0899 & 0.3200 & 0.0233 & 0.0283 & 0.0207 \\
\hline Breas & 6 & 027 & 0031 & 0.0030 & 0.0053 & 0.0035 & 0037 & 0042 & 0.0048 & 0.0053 & 0.0057 & 0.0064 & 0.0066 & 0.0067 & 0.0077 & 0.0272 & 0036 & 0.0040 & .0033 \\
\hline Gallbladder wall & 0.0107 & 084 & 0.0093 & 0.0092 & 0.0147 & 0.0109 & 0.0105 & 0.0121 & 0.0135 & 0.0147 & 0.0158 & 0.0174 & 0.0180 & 0.0189 & 0.0211 & 0.0744 & 0110 & 0.0124 & .0096 \\
\hline ver large int & (2021 & & & 0.0026 & & 0.0031 & 0.0032 & 0.0036 & 0.0040 & 0.0044 & 0.0048 & 0.0053 & 0.0055 & 0.0058 & 0.0066 & & & & \\
\hline $\mathrm{Sm}$ & 0.0041 & 032 & 036 & 0.0035 & 0.0057 & 0.0041 & 0.0041 & 0.0047 & 0.0052 & 0.0057 & 0.0062 & 0.0067 & 0.0071 & 0.0075 & 0.0084 & 0.0296 & 0043 & 0.0048 & 0038 \\
\hline$c_{0}$ & 1060 & & 9947 & 0.0937 & 0.1230 & 0.1110 & 0.1010 & 0.1090 & 0.1160 & 0.1230 & 0.1300 & 0.1420 & 0.1440 & 0.1560 & 0.1820 & 0.6270 & .0993 & & .0925 \\
\hline$r$ lower intest & 0.0046 & 036 & 0040 & 0.0040 & 0.0063 & 0.0047 & 0.0046 & 0.0052 & 0.0058 & 0.0063 & 0.0069 & 0.0075 & 0.0078 & 0.0083 & 0.0093 & 0.0327 & 0048 & 0.0 & 0.0043 \\
\hline & 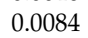 & & 072 & 0.0071 & 23 & 0.0084 & 0086 & 0.0097 & 0.0112 & 0.0123 & 0.0133 & 0.0148 & 0.0152 & 0.0155 & 0.0179 & & 36 & & 0.0077 \\
\hline K & 89 & & 650 & 0.1570 & 0.2680 & 0.1870 & 0.1800 & 0.2130 & 0.2420 & 0.2680 & 0.2930 & 0.2850 & 0.3390 & 0.3600 & 0.4040 & 1.4100 & 1830 & 0.2030 & 0.1720 \\
\hline Liver & 9944 & 746 & 0828 & 0.0821 & 0.1300 & 0.0979 & 0.0924 & 0.1070 & 0.1190 & 0.1300 & 0.1390 & 0.1550 & 0.1580 & 0.1660 & 0.1810 & 0.6440 & 0990 & 0.1130 & 0.0840 \\
\hline & & & & 0.1390 & & & & & 0.2360 & 0 & 0.2800 & & 0.3200 & 0.3220 & 0.3750 & & & & .1530 \\
\hline Muscle & 0.0035 & 27 & 030 & 0.0030 & 0.0051 & 0.0035 & 0.0035 & 0.0041 & 0.0046 & 0.0051 & 0.0055 & 0.0061 & 0.0063 & 0.0066 & 0.0075 & 0.0264 & 0037 & 0.0040 & .0033 \\
\hline Orurion & & & 9027 & 0.0026 & & 00032 & & 0.0037 & & 0.0045 & & 0.0054 & 0.0057 & 0.0060 & 0.0067 & & & & 0.0031 \\
\hline Pancreas & 07 & & 0143 & 0.0141 & 0.0228 & 0.0162 & 0.0163 & 0.0187 & 0.0208 & 0.0228 & 0.0248 & 0.0276 & 0.0287 & 0.0297 & 0.0346 & 0.1210 & 0.0174 & 0.0190 & 0.0154 \\
\hline Red. Ma & 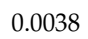 & & 0033 & 0.0032 & & 0.0037 & & 0.0044 & 0.0051 & 0.0056 & 0.0062 & 0.0068 & 0.0071 & 0.0074 & 0.0084 & 0.0296 & 0.0039 & 0.0 & 0.0035 \\
\hline Ostec & c & & 26 & 0.0026 & 7 & 0.0030 & & 0.0036 & 0.0042 & 0.0047 & 0.0051 & 0.0057 & 0.0059 & 0.0061 & 0.0070 & 0.0245 & .0031 & 0.0035 & 0.0028 \\
\hline $\mathrm{Sk}$ & 0017 & 013 & 0015 & 0.0014 & 0.0025 & 0.0017 & 0.0017 & 0.0020 & 0.0023 & 0.0025 & 0.0027 & 0.0030 & 0.0032 & 0.0033 & 0.0037 & 0.0131 & 0.0018 & 0.0019 & 0.0016 \\
\hline & & & & 03820 & & & & 0.5380 & 0.6040 & 0.6730 & 0.7460 & 0.8620 & 0.8940 & 0.8990 & 1.0900 & & 5390 & 0.5960 & 0.4590 \\
\hline Thymus & o & 9 & 0.0033 & 0.0032 & 0.0058 & 0.0038 & 0.0040 & 0.0045 & 0.0053 & 0.0058 & 0.0063 & 0.0070 & 0.0072 & 0.0073 & 0.0084 & 0.0296 & 0.0039 & 0.0042 & 0.0035 \\
\hline & & & & 0.0011 & & 0.0013 & 0.0014 & 0.0016 & 0.0019 & 0.0021 & 0.0023 & 0.0026 & 0.0027 & 0.0027 & 0.0031 & 0.0110 & 0.0013 & 0.0015 & 0.0012 \\
\hline Urinary bla & & & & 0.1 & & & & & & 0.2 & & 0.2890 & 0.2 & & 0.3500 & & 0.1870 & 0.2120 & 0.1620 \\
\hline Uterus & 0.0050 & 0.0037 & 0.0042 & 0.0042 & 0.0073 & 0.0050 & 0.0052 & 0.0060 & 0.0067 & 0.0073 & 0.0080 & 0.0089 & 0.0092 & 0.0097 & 0.0108 & 0.0381 & 0.0057 & 0.0064 & 0.0050 \\
\hline
\end{tabular}




\section{Discussion}

Radiation dosimetry provides information on the absorption of energy from ionizing radiation and the effect of radiation damage to living tissues during immuno-PET. Dosimetry can be used to provide data on the safety and efficacy of immuno-PET $[9,10]$.

In this study, we investigated the effect of reconstruction algorithms and filters on radiation dosimetry for Cu-64 trastuzumab PET. For this purpose, image quality parameters, such as NU, RC, and SOR, were assessed for various reconstruction algorithms implemented in Siemens Inveon PET/CT. We suggested selection criteria for determining the optimal reconstruction algorithm and filter for the dosimetry study of $\mathrm{Cu}-64$ trastuzumab PET.

\subsection{Spill-Over Ratio}

For both the air and water compartments, there were negative activity values in FBP or FBP-3DRP analytical algorithms but not in OSEM 2D or OSEM3D-MAP statistical iterative algorithms. FBP or FBP-3DRP could provide negative values because of ramp filtering [22]. The lowest SOR values were generally associated with the analytic algorithms because they can trigger negative background activity values and thus negative SOR scores. The OSEM-based OSEM2D and OSEM3D-MAP algorithms are characterized by the so-called non-negativity constraint and thus cannot yield negative SOR scores [23].

\subsection{Selection of the Optimal Reconstruction Algorithm (a Trade-Off Relationship between Recovery Coefficients} and Non-Uniformity)

To determine the optimal reconstruction algorithm, graphs between RC and NU were illustrated for partially corrected, AC, and both AC and SC data sets (Figure 3a-c). The factors that determine $\mathrm{RC}$ include the size of the lesion. The factors determining the spatial resolution in the image are the matrix size of the image, the image reconstruction method, and the filter [24]. The NU is an index that measures the uniformity of an image. RC can be considered as an index for the measurement of the peak value of a tumor. NU was the parameter for the assessment of the warm background around the tumor. The process of detecting a tumor in PET is the process of detecting a hot region on a warm background. Therefore, it is reasonable to consider RC and NU at the same time. In this study, we assumed that the optimal reconstruction algorithm should satisfy the condition $0.9<\mathrm{RC}<1.0$ and $\mathrm{NU}<10 \%$ simultaneously. The optimal criteria were marked in red color in Figure 3a,b. A high NU value means a noisy background in PET data. The tumor detectability would decrease in case of a high NU. According to the optimal criteria $(0.9<\mathrm{RC}<1.0$ and $\mathrm{NU}<10 \%)$ in the present study, OSEM2D (iteration 1) provided an optimal image parameter with an RC near 1 and low NU for both partially corrected and AC images. OSEM2D (iteration 1) provided an RC value of 0.92 and an NU of $7.0 \%$ for partially corrected and RC of 0.94 and NU of 7.1\% for AC, respectively. However, when the AC and SC were applied, the RC value deteriorated to 0.70 for OSEM2D (iteration 1).

\subsection{Effect of Attenuation Correction and Scatter Correction}

In this study, we focused on the correlation between NU and RC when no AC and/or AC/SC were performed. This was because AC and/or AC/SC was omitted during PET scanning of the mice When AC or AC/SC was performed, the tendency was similar to that of the partially corrected.

\subsection{Correlation between Recovery Coefficient and \% ID/g in the Tumor Region}

To investigate whether the RC value calculated for phantom correlates with \% ID/g calculated for $\mathrm{Cu}-64$ trastuzumab, the $\mathrm{RC}$ value in this study was calculated for various reconstruction algorithms using the NEMA NU4 image quality phantom. RC from phantom study and \% ID/g from $\mathrm{Cu}-64$ trastuzumab PET were compared. For direct comparison with RC and \% ID/g, RC and \% ID/g were normalized with a maximum value of $\mathrm{RC}$ and $\% \mathrm{ID} / \mathrm{g}$, respectively. The graphs of normalized RC and normalized \% ID/g were plotted for various reconstruction algorithms. The ROI size for the tumor 
region was $0.066 \mathrm{~cm}^{2}$ (equivalent to a $3 \mathrm{~mm}$ rod for the $\mathrm{RC}$ phantom). Figure $6 \mathrm{~b}$ shows the correlation between RC and \% ID/g in the tumor region. Normalized RC and \% ID/g in the tumor region were highly correlated $\left(R^{2}=0.97\right)$. RC was correlated with a value such as $\% \mathrm{ID} / \mathrm{g}$ or standard uptake value (SUV) on actual PET data sets. Thus, we could apply the optimal reconstruction algorithm, as determined from the proposed criteria in this study, for the actual calculation of dosimetry for Cu-64 trastuzumab PET.

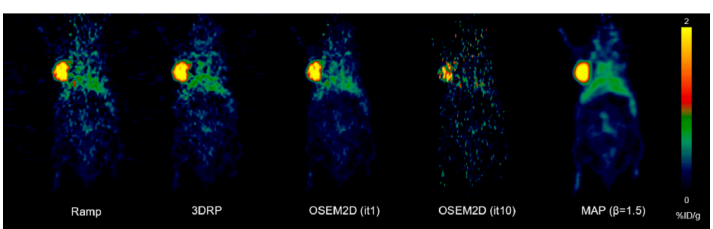

(a)

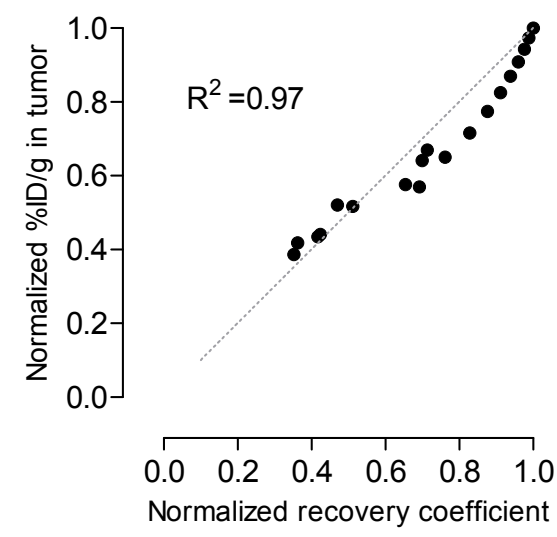

(c)

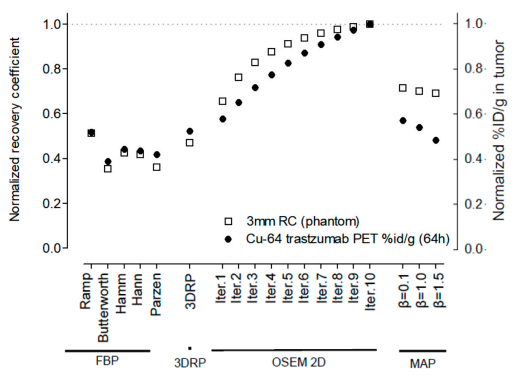

(b)

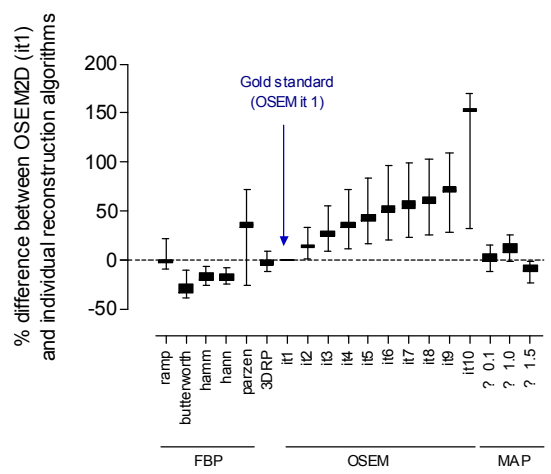

(d)

Figure 6. (a) Representative \% ID/g image of Cu-64 trastuzumab PET using FBP (ramp filter), FBP-3DRP, OSEM2D (iteration 1), OSEM2D (iteration 10), and OSEM3D-MAP ( $\beta=1.5$ ). (b) Plot of normalized recovery coefficient (rod diameter is $3 \mathrm{~mm}$ ) and normalized \% ID/g in the tumor on Cu-64 trastuzumab PET. The recovery coefficient and \% ID/g were normalized using each maximum value for comparison. (c) A plot of correlation between the normalized recovery coefficient (rod diameter is $3 \mathrm{~mm}$ ) and the normalized \% ID/g. The parameter was highly correlated $\left(\mathrm{R}^{2}=0.97\right)$. (d) \% difference between OSEM2D (iteration 1, the gold standard in this study) and individual reconstruction algorithms. The data was plotted using the averaged value of the difference for all organs and tumor regions. The data was plotted using the box and whisker plot. 3DRP: FBP-3DRP, MAP: OSEM3D-MAP.

\subsection{Optimal Reconstruction Algorithm and Tumor Absorbed Dose}

Table 1 presents the results of tumor dosimetry. According to the results, the value of the absorbed dose for the tumor ranged from $923 \mathrm{mGy} / \mathrm{MBq}$ to $1830 \mathrm{mGy} / \mathrm{MBq}$. The difference between the maximum and minimum absorbed dose reached $98 \%$. The minimum value of the absorbed dose for the tumor was obtained when FBP was used with Butterworth filter; the maximum value of the absorbed dose for a tumor was obtained when using OSEM2D with 10 iterations and 16 subsets.

Table 2 presents the \% difference between OSEM2D (iteration 1, the gold standard in this study) and individual reconstruction algorithms. When OSEM2D (iteration 1) was chosen as the optimal reconstruction algorithm based on the phantom study in this work (Figure 3a), OSEM2D (iteration 10) yielded 32.4\% overestimated absorbed dose and FBP with Butterworth filter yielded $35.4 \%$ underestimated absorbed dose for the tumor. The maximum difference is marked in bold. 
Table 2. \% difference between OSEM2D (iteration 1, gold standard in this study) and individual reconstruction algorithms.

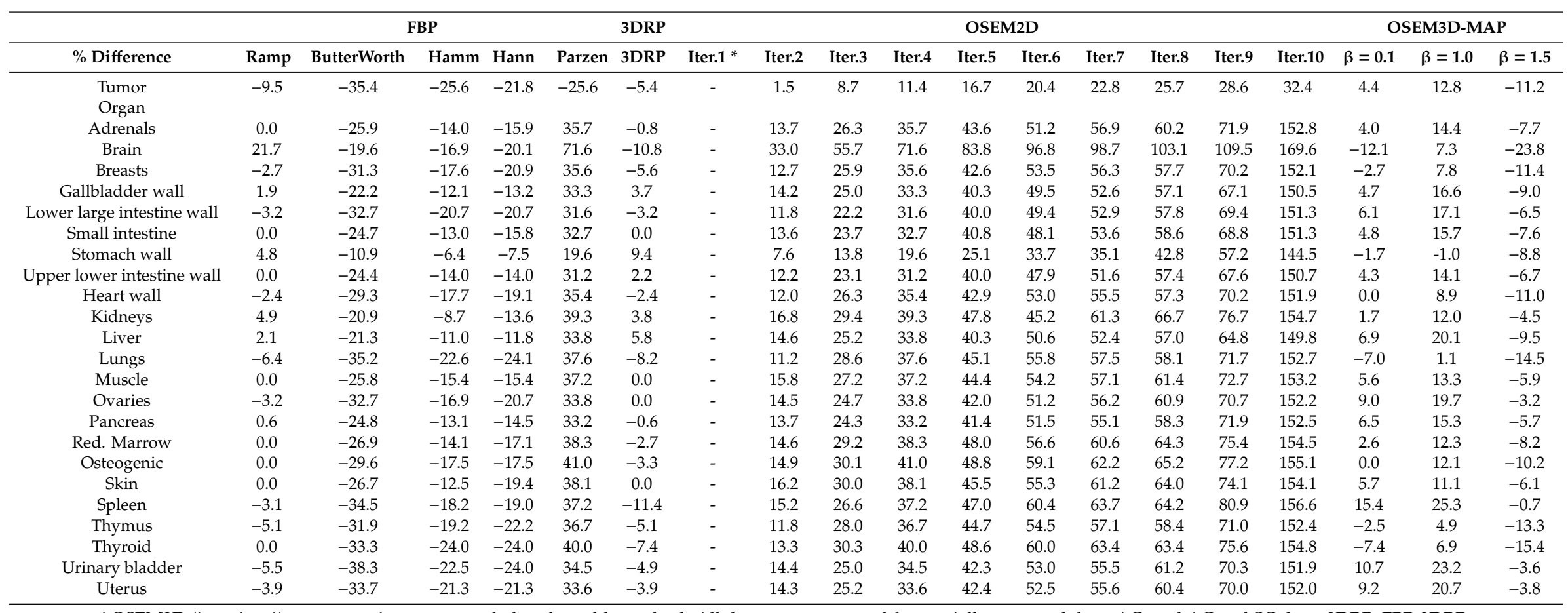

* OSEM2D (iteration 1) reconstruction was regarded as the gold standard. All data were presented for partially corrected data, AC, and AC and SC data. 3DRP: FBP-3DRP. 
There is the same tendency for all organs. For example, in the region of brain, OSEM2D (iteration 10) yielded $152.8 \%$ overestimated effective dose and FBP with the Butterworth filter provided $25.9 \%$ underestimated effective dose. In the region of the kidney, the difference was $154.7 \%$ overestimation for OSEM2D (iteration 10) and 20.9\% underestimation for FBP with Butterworth filter.

Figure $6 \mathrm{c}$ presents the averaged values of the \% difference between OSEM2D (iteration 1, the gold standard in this study) and individual reconstruction algorithms. The box and whisker plot was used for Figure 6c. According to Figure $6 c$, we also found that there is a large difference between OSEM2D (iteration 1) and OSEM2D (iteration 10).

The limitation of this study is the potential bias of individual difference in dosage. This study essentially describes image quality characteristics as a function of reconstruction parameters for $\mathrm{Cu}-64$. The data shown in Figure $6 \mathrm{c}$ were obtained from a single tumor uptake value. Therefore, the range in values represented was only due to differences in reconstruction.

\section{Conclusions}

OSEM2D (iteration 1) was determined for optimal reconstruction algorithms for minimizing the non-uniformity and for obtaining the highest RC. We suggested OSEM2D (iteration 1) for the calculation of the effective dose of Cu-64 trastuzumab on an Inveon PET scanner.

Author Contributions: Conceptualization, J.S.K.; methodology, J.S.K.; software, S.L.; validation, J.M.K.; formal analysis, S.L., Y.-r.K., and H.K. (Hyeongi Kim); investigation, S.L. and H.K. (Hyungwoo Kim); resources, Y.-J.L.; data curation, S.L. and J.Y.K.; writing-original draft preparation, S.L.; writing-review and editing, J.S.K.; visualization, S.L. and H.K. (Hyeongi Kim).; supervision, J.S.K.; project administration, J.S.K.; funding acquisition, J.S.K. and Y.-J.L.

Funding: This study was supported by a grant of the Korea Institute of Radiological and Medical Sciences (KIRAMS), funded by Ministry of Science and ICT (MSIT), Korea. (No.50461-2019, PI: Kyo Chul Lee and 50536-2019, PI: Y.-J.L.) and supported by the Ministry of Health and Welfare (No HO15C0003, PI: J.S.K.)

Acknowledgments: We thank you for providing Cu-64 for research at the cyclotron center at KIRAMS. We also thank Shin-Hye Kwak for her experimental support.

Conflicts of Interest: The authors declare no conflict of interest. The funders had no role in the design of the study; in the collection, analyses, or interpretation of data; in the writing of the manuscript, or in the decision to publish the results.

\section{Appendix A}

\section{Appendix A.1. Spatial Resolution}

To measure the spatial resolution, $1 \mathrm{~mm}^{3}$ sized point source (inner diameter $1.1 \mathrm{~mm}$; wall thickness $0.2 \mathrm{~mm}$ ) of Cu- 64 was used. PET data was acquired for 5 min within an energy window of $\sim 350-650 \mathrm{keV}$ and a timing window of $3.432 \mathrm{~ns}$. The list mode data were sorted into 3D sinograms and then reconstructed with various parameters. To determine the spatial resolution in radial, tangential, and axial directions, profiles through peaks in count distributions were drawn in two orthogonal directions in middle slices. For each profile, the full width at half maximum (FWHM) was measured by linear interpolation between adjacent pixels at one half or one tenth of their maximum values in profile. Axial profiles were also drawn to measure axial resolution. Volumetric resolution was calculated by multiplying resolutions for radial, tangential, and axial directions.

\section{Appendix A.2. The Effect of Reconstruction Algorithms with Various Filters}

The effect of reconstruction algorithms was assessed for the FBP, 2D ordered subset expectation maximization (OSEM 2D), 3D reprojection algorithm (FBP-3DRP), and OSEM maximum a posteriori (OSEM3D-MAP) algorithms. In addition, the effect of filters in FBP was assessed for Butterworth, Hamming, Hanning, Parzen, ramp (cut off frequency: 0.5) and Shepp-Logan filters. For OSEM2D, the result of various iteration number from 1 to 10 was also assessed for the measurement of the effect of iteration number. Subset number was fixed to 16 for OSEM2D. For OSEM3D-MAP reconstruction, 
various smoothing factors $(\beta)(0.1,1.0,1.5)$ were used with iteration number 18 . The pixel size was 0.26 $\times 0.26 \mathrm{~mm}^{2}$. Normalization was applied, whereas AC and SC were not applied. The slice thickness values in all reconstructed images were $0.796 \mathrm{~mm}$. Figure A1a,b shows the effect of reconstruction algorithms and filters on image resolution. The highest achievable volumetric resolution was $3.07 \mathrm{~mm}^{3}$ and it was obtained when OSEM3D-MAP $(\beta=0.1)$ was used.
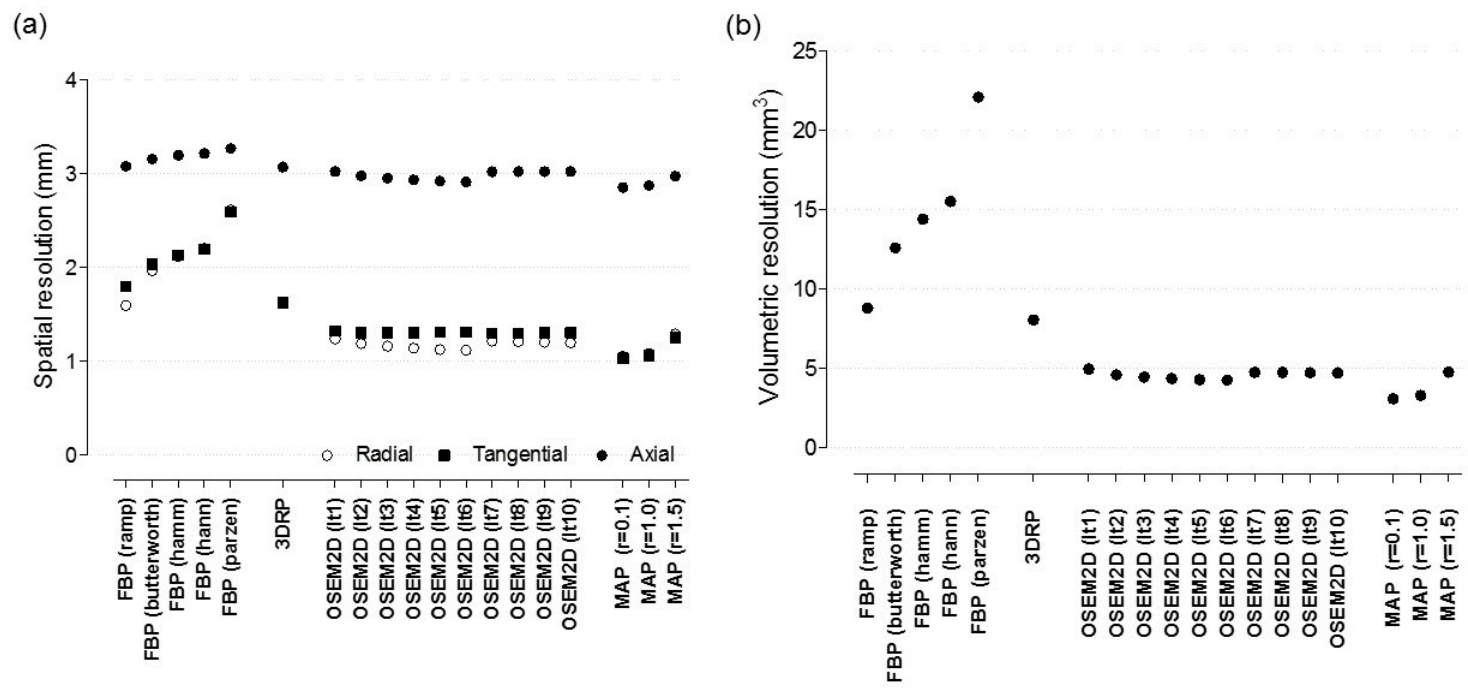

Figure A1. Spatial resolution of Cu-64 (a) radial, tangential, axial spatial resolution, (b) volumetric spatial resolution, 3DRP: FBP-3DRP, MAP: OSEM3D-MAP.

Appendix A.3. The Effect of Post-Smoothing with Various Gaussian Kernel

Figure A2 shows the result of OSEM2D (iteration 1) and OSEM3D-MAP ( $\beta=1.5)$ data with application of Gaussian smoothing kernel. $1.5 \mathrm{~mm}, 3.0 \mathrm{~mm}, 4.5 \mathrm{~mm}$, and $6.0 \mathrm{~mm}$ FWHM Gaussian kernel were applied to reconstructed PET data. Figure A2a,c show the plots of NU vs. RC (rod diameter is $3 \mathrm{~mm}$ ) for OSEM2D (iteration 1) and OSEM3D-MAP ( $\beta=1.5$ ) with Gaussian kernel smoothed respectively. Figure A2b,d show PET images with Gaussian smoothing of phantom with OSEM2D (iteration 1) and OSEM3D-MAP $(\beta=1.5)$ reconstruction respectively. There was a tendency to decrease RC and NU as Gaussian smoothing kernel was applied. The percent difference of RC between without and with $6.0 \mathrm{~mm}$ Gaussian kernel smoothed was in $82 \%$ for OSEM2D (iteration 1) and $79 \%$ for OSEM3D-MAP ( $\beta=1.5)$. The percent difference of NU between without and with $6.0 \mathrm{~mm}$ Gaussian smoothing was $68 \%$ for OSEM2D (iteration 1 ) and $69 \%$ for OSEM3D-MAP $(\beta=1.5)$. 
(a)

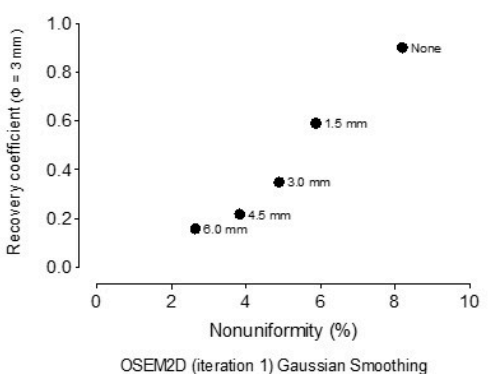

(c)

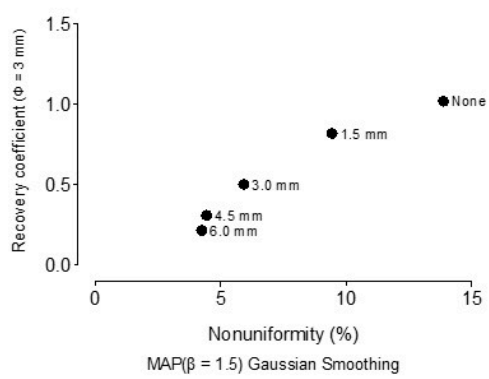

(b)

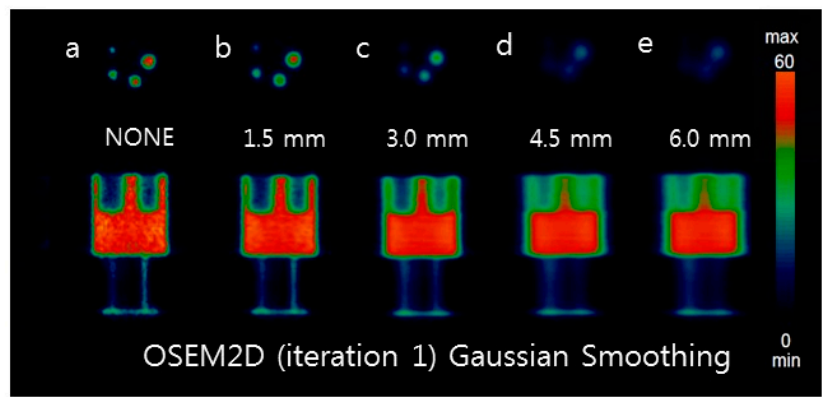

(d)

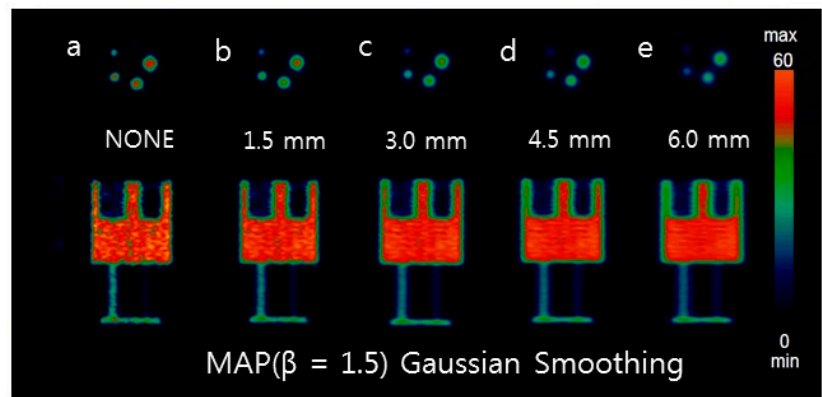

Figure A2. RC and NU of OSEM2D (iteration 1) and OSEM3D-MAP ( $\beta=1.5)$ data with application of Gaussian smoothing kernel. Applied Gaussian FWHM was $1.5 \mathrm{~mm}, 3.0 \mathrm{~mm}, 4.5 \mathrm{~mm}$, and $6.0 \mathrm{~mm}$, respectively. (a) Plot of NU vs. RC (rod diameter, $3 \mathrm{~mm}$ ) for OSEM2D (iteration 1), (b) representative PET data, (c) plot of NU vs. RC (rod diameter, $3 \mathrm{~mm}$ ) for OSEM3D-MAP $(\beta=1.5)$, and (d) representative PET data. MAP: OSEM3D-MAP.

\section{Appendix A.4. Comparison between Reconstructed Data and Bio-Distribution Data}

To investigate the correlation between reconstructed data and bio-distribution data, we additionally performed PET and in vivo biodistribution study using Cu-64 DOTA-anti-PD-1 antibody data. Figure $\mathrm{A} 3$ shows the PET of Cu-64 DOTA-anti-PD-1-antibody and the correlation data reconstructed data and bio-distribution data. To acquire the PET/CT images, the Cu-64 DOTA-anti-PD-1-antibody (17.39-20.35 MBq, $400 \mu \mathrm{g}$ ) was administered to Hap-T1 cell-bearing hamsters when the tumor size reached $89.1-103 \mathrm{~mm}^{3}$. The immuno-PET/CT scanning (Siemens Inveon) was performed with an acquisition time of $15 \mathrm{~min}$ within an energy window of 350 to $650 \mathrm{keV}$ at $60 \mathrm{~h}$ after injection of $\mathrm{Cu}-64$ DOTA-anti-PD-1-Ab. In vivo biodistribution studies were also performed to evaluate the true uptake of $\mathrm{Cu}-64$ DOTA-anti-PD-1-Ab in hamsters. Immediately after PET scanning, hamsters $(n=3)$ were euthanized by $\mathrm{CO}_{2}$ gas asphyxiation. The tumors were collected and weighed. The radioactivity of the tumor was measured as the total number of counts (counts per minute, $\mathrm{cpm}$ ) using a Wizard2 gamma-counter (PerkinElmer). The activity data were represented as the percentage injected radioactivity dose per gram of tissue (\%ID/g). PET data were reconstructed using various reconstruction algorithms and filters. \%difference error between reconstructed data and bio-distribution data was calculated as follows: \%difference error $=(\% \mathrm{ID} / \mathrm{g}$ of reconstructed data $-\mathrm{D} / \mathrm{g}$ of bio-distribution data)/\%ID/g of bio-distribution data. $\%$ difference error of OSEM (it $=1$ ) ranged from $-1.5 \%$ to $-47.3 \%$. This meant that the reconstructed value was lower than that of the bio-distribution data. When OSEM3D-MAP reconstruction method was used, the \%difference error ranged from $-13 \%$ to $28 \%$. The positive value of the \%difference error meant that the value of reconstructed data was higher than that of the bio-distribution data. This phenomenon was also observed in phantom data. This higher value was because of Gibbs artifact during OSEM3D-MAP reconstruction [25]. We found that the $\% \mathrm{ID} / \mathrm{g}$ of reconstructed PET data was lower than that of actual bio-distribution data. This phenomenon has also been found in a previous report [25]. According to the previous report, \%ID/g of PET data was degraded by $20.9 \%$ compared to bio-distribution data [26]. This difference was possibly due to the effect of the partial volume on PET data. Because of limited spatial resolution on PET, the partial volume effect on the image of a tumor can degrade PET count. 
Another distinction is background uniformity. On the reconstructed PET data, image contrast would be important to detect tumor site. RC and NU would be deterministic factors in terms of detectability and image contrast. However, bio-distribution was free from partial volume effect and/or background activity because tumor or only the region being investigated was extracted and \% ID/g was measured during bio-distribution experiment.

(a)

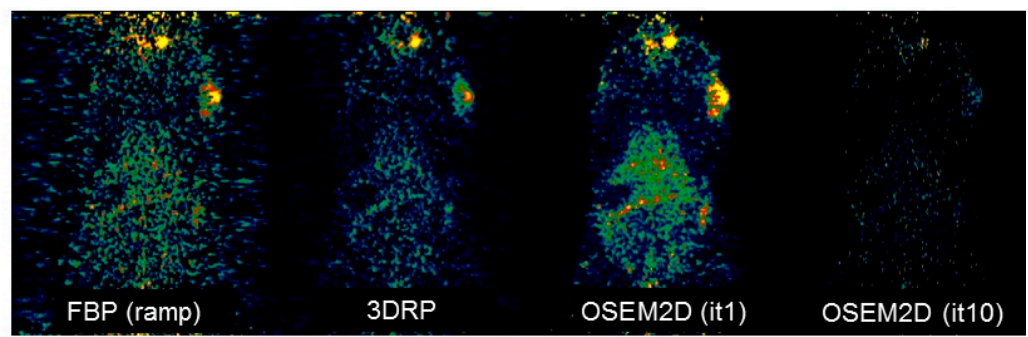

(b)

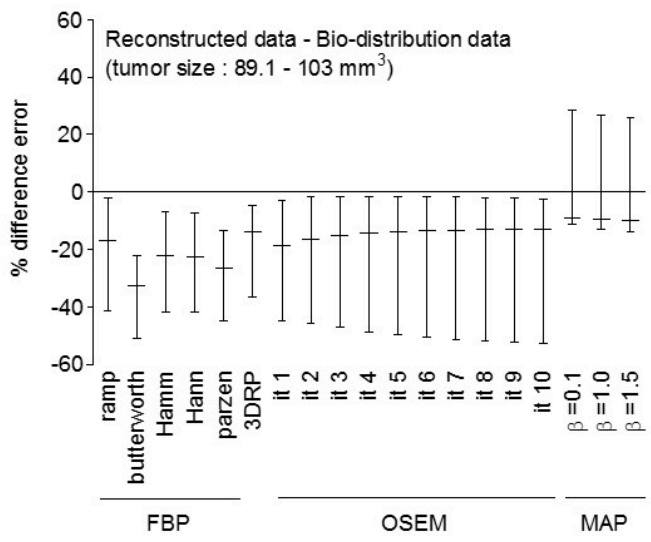

Figure A3. (a) representative PET of Cu-64 DOTA-anti-PD-1-antibody with FBP (ramp Figure 3. OSEM2D (it 1), OSEM2D (it 10), (b) \%difference between reconstructed data and bio-distribution data. 3DRP: FBP-3DRP, MAP: OSEM3D-MAP.

Appendix A.5. Spill-Over Ratio vs. Recovery Coefficient

In this study, to determine the optimal reconstruction algorithm reconstruction, graphs between RC and NU were illustrated. In addition, we also plotted the graph between SOR (air, water) and the recovery coefficient in Figure A4a,b. SOR for water was $>18 \%$ for all reconstruction algorithms and filters. RC was 0.92 and SOR for water was $26.4 \%$ when OSEM2D (it 1) was used. 
(a)

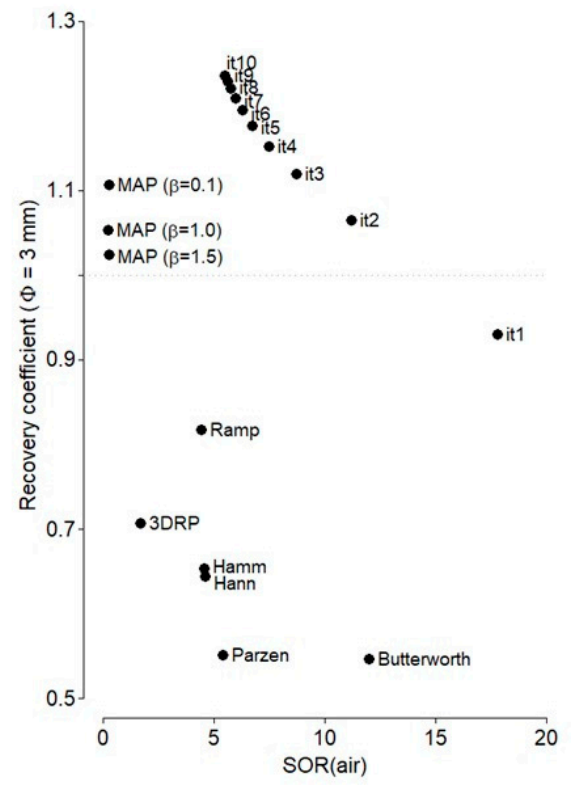

(b)

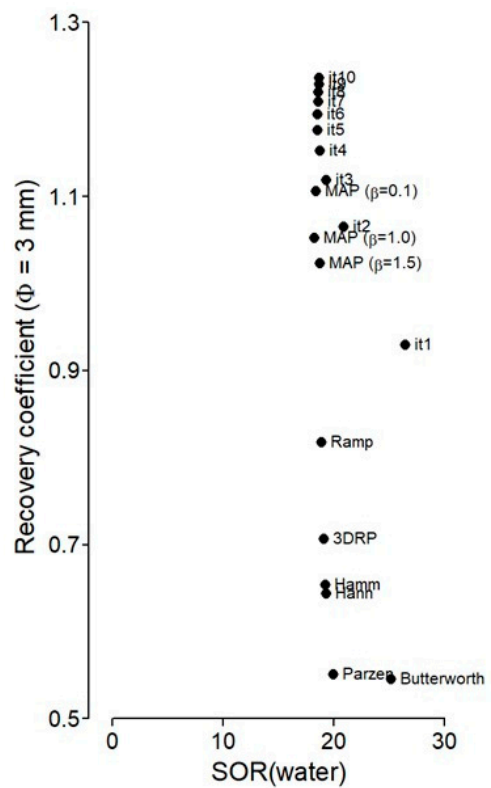

Figure A4. (a) SOR (air) vs. RC, (b) SOR (water) vs. RC. 3DRP: FBP-3DRP, MAP: OSEM3D-MAP.

Appendix A.6. Assessment of Zr-89 PET Data in Terms of Spatial Resolution and SOR and NU

$\mathrm{Zr}-89$ was also used for immune-PET. Therefore, we also assessed the spatial resolution and the relationship between the recovery coefficient and non-uniformity. The experimental procedures were the same as that of $\mathrm{Cu}-64$.

Appendix A.7. The effect of Reconstruction Algorithms with Various Filters

Figure A5a,b shows the result of spatial resolution. The highest achievable volumetric resolution was $3.09 \mathrm{~mm}^{3}$ and it was obtained when OSEM3D-MAP $(\beta=0.1)$ was used.

(a)

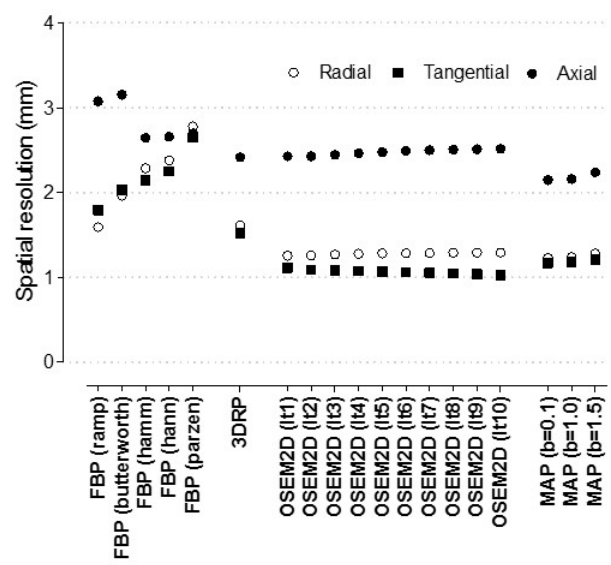

(b)

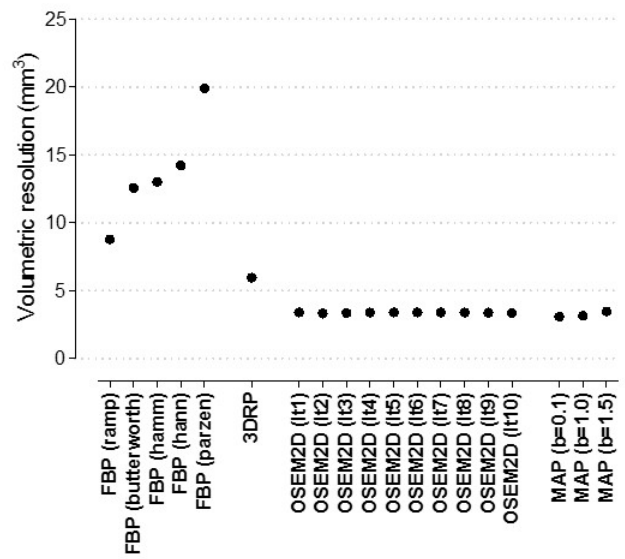

Figure A5. Spatial resolution of Zr-89 (a) radial, tangential, and axial spatial resolution, (b) volumetric spatial resolution. 3DRP: FBP-3DRP, MAP: OSEM3D-MAP.

Appendix A.8. Spill-Over Ratio vs. Non-Uniformity

Figure A6a-c shows $\mathrm{RC}$ for the $3 \mathrm{~mm}$ rods plotted against the non-uniformity when partially corrected, AC, and AC and SC, respectively. There was the same tendency between Cu-64 and Zr- 89 . 

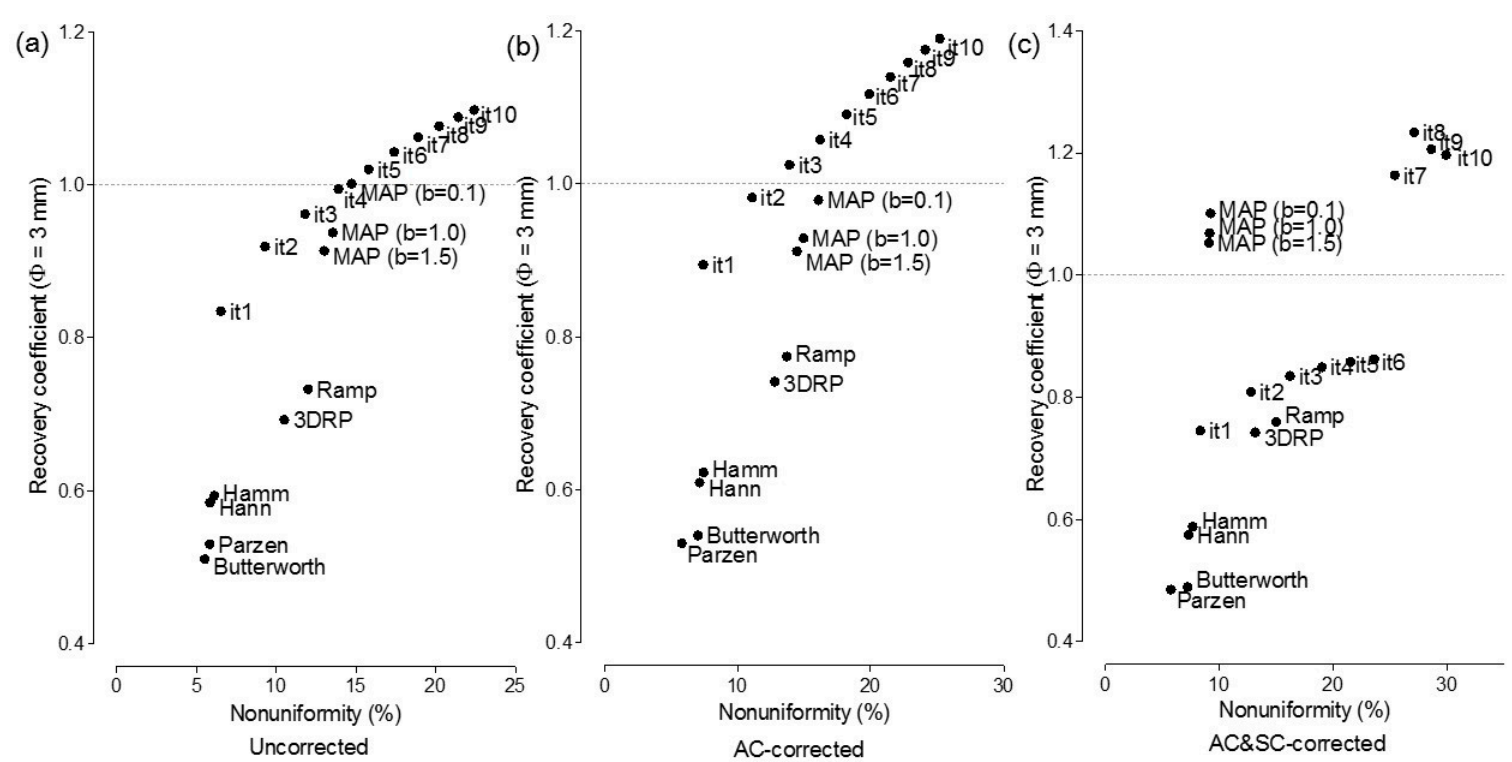

Figure A6. Plot of non-uniformity versus recovery coefficient (rod diameter is $3 \mathrm{~mm}$ ) for various reconstruction algorithms, such as FBP, FBP-3DRP, OSEM2D, and OSEM3D-MAP reconstruction with filters. All data were presented for (a) partially corrected data, (b) AC, and (c) AC and SC data. 3DRP: FBP-3DRP, MAP: OSEM3D-MAP.

\section{References}

1. Boerman, O.C.; Oyen, W.J. Immuno-PET of cancer: A revival of antibody imaging. J. Nucl. Med. 2011, 52, 1171-1172. [CrossRef]

2. Kim, J.S. Combination Radioimmunotherapy Approaches and Quantification of Immuno-PET. Nucl. Med. Mol. Imaging 2016, 50, 104-111. [CrossRef] [PubMed]

3. Mestel, R. Cancer: Imaging with antibodies. Nature 2017, 543, 743-746. [CrossRef]

4. van Dongen, G.A.; Visser, G.W.; Lub-de Hooge, M.N.; de Vries, E.G.; Perk, L.R. Immuno-PET: A navigator in monoclonal antibody development and applications. Oncologist 2007, 12, 1379-1389. [CrossRef]

5. Tamura, K.; Kurihara, H.; Yonemori, K.; Tsuda, H.; Suzuki, J.; Kono, Y.; Honda, N.; Kodaira, M.; Yamamoto, H.; Yunokawa, M.; et al. Cu-64 DOTA-trastuzumab PET imaging in patients with HER2-positive breast cancer. J. Nucl. Med. 2013, 54, 1869-1875. [CrossRef]

6. Mortimer, J.E.; Bading, J.R.; Colcher, D.M.; Conti, P.S.; Frankel, P.H.; Carroll, M.I.; Tong, S.; Poku, E.; Miles, J.K.; Shively, J.E.; et al. Functional imaging of human epidermal growth factor receptor 2-positive metastatic breast cancer using Cu-64 DOTA-trastuzumab PET. J. Nucl. Med. 2014, 55, 23-29. [CrossRef] [PubMed]

7. Kurihara, H.; Hamada, A.; Yoshida, M.; Shimma, S.; Hashimoto, J.; Yonemori, K.; Tani, H.; Miyakita, Y.; Kanayama, Y.; Wada, Y.; et al. Cu-64 DOTA-trastuzumab PET imaging and HER2 specificity of brain metastases in HER2-positive breast cancer patients. EJNMMI Res. 2015, 5, 8. [CrossRef]

8. Woo, S.K.; Jang, S.J.; Seo, M.J.; Park, J.H.; Kim, B.S.; Kim, E.J.; Lee, Y.J.; Lee, T.S.; An, G.I.; Song, I.H.; et al. Development of Cu-64 -NOTA-Trastuzumab for HER2 targeting: Radiopharmaceutical with improved pharmacokinetics for human study. J. Nucl. Med. 2018, 60, 26-33. [CrossRef] [PubMed]

9. Stabin, M.G.; Sparks, R.B.; Crowe, E. OLINDA/EXM: The second-generation personal computer software for internal dose assessment in nuclear medicine. J. Nucl. Med. 2005, 46, 1023-1027.

10. Lee, Y.S.; Kim, J.S.; Cho, K.D.; Kang, J.H.; Lim, S.M. Tumor dosimetry for I-131 trastuzumab therapy in a Her2+ NCI N87 xenograft mouse model using the Siemens SYMBIA E gamma camera with a pinhole collimator. J. INSTRUM 2015, 10, P07001. [CrossRef]

11. Poston, J.W. Application of the effective dose equivalent to nuclear medicine patients. The MIRD Committee. J. Nucl. Med. 1993, 34, 714-716.

12. Howell, R.W. The MIRD Schema: From organ to cellular dimensions. J. Nucl. Med. 1994, 35, 531-533. 
13. Boellaard, R.; Delgado-Bolton, R.; Oyen, W.J.; Giammarile, F.; Tatsch, K.; Eschner, W.; Verzijlbergen, F.J.; Barrington, S.F.; Pike, L.C.; Weber, W.A.; et al. FDG PET/CT: EANM procedure guidelines for tumour imaging: Version 2.0. Eur. J. Nucl. Med. Mol. Imaging 2015, 42, 328-354. [CrossRef]

14. NEMA. Performance Measurements of Small Animal Positron Emission Tomographs (PETs). In NEMA Standards Publication NU 4-2008; NEMA: Rosslyn, VA, USA, 2008.

15. Bahri, M.A.; Plenevaux, A.; Warnock, G.; Luxen, A.; Seret, A. NEMA NU4-2008 image quality performance report for the microPET focus 120 and for various transmission and reconstruction methods. J. Nucl. Med. 2009, 50, 1730-1738. [CrossRef]

16. Constantinescu, C.C.; Mukherjee, J. Performance evaluation of an Inveon PET preclinical scanner. Phys. Med. Biol. 2009, 54, 2885-2899. [CrossRef]

17. Bao, Q.; Newport, D.; Chen, M.; Stout, D.B.; Chatziioannou, A.F. Performance evaluation of the inveon dedicated PET preclinical tomograph based on the NEMA NU-4 standards. J. Nucl. Med. 2009, 50, 401-408. [CrossRef]

18. Lasnon, C.; Dugue, A.E.; Briand, M.; Blanc-Fournier, C.; Dutoit, S.; Louis, M.H.; Aide, N. NEMA NU 4-Optimized Reconstructions for Therapy Assessment in Cancer Research with the Inveon Small Animal PET/CT System. Mol. Imaging Biol. 2015, 17, 403-412. [CrossRef]

19. Bradshaw, T.J.; Voorbach, M.J.; Reuter, D.R.; Giamis, A.M.; Mudd, S.R.; Beaver, J.D. Image quality of Zr-89 PET imaging in the Siemens microPET Focus 220 preclinical scanner. Mol. Imaging Biol. 2016, 18, 377-385. [CrossRef]

20. Yu, A.R.; Kim, J.S. Effect of filters and reconstruction algrorithms on I-124 PET in Siemens Inveon PET scanner. J. INSTRUM 2015, 10, P10026. [CrossRef]

21. Laforest, R.; Lapi, S.E.; Oyama, R.; Bose, R.; Tabchy, A.; Marquez-Nostra, B.V.; Burkemper, J.; Wright, B.D.; Frye, J.; Frye, S.; et al. Zr-89 Trastuzumab: Evaluation of Radiation Dosimetry, Safety, and Optimal Imaging Parameters in Women with HER2-Positive Breast Cancer. Mol. Imaging Biol. 2016, 18, 952-959. [CrossRef]

22. O'Sullivan, F.; Pawitan, Y.; Haynor, D. Reducing negative artifact in emission tomography. IEEE Trans. Med. Imaging 1993, 12, 653-663. [CrossRef] [PubMed]

23. Hudson, H.M.; Larkin, R.S. Accelerated image reconstruction using ordered subsets of projection data. IEEE Trans. Med. Imaging 1994, 13, 601-619. [CrossRef] [PubMed]

24. Lee, Y.S.; Kim, J.S.; Kim, H.-J.; Woo, S.K.; Kim, J.G.; Park, J.A.; Choi, C.W.; Lim, S.M.; Kim, K.M. Imaging Characteristics of I-124 Between 3D and 2D on Siemens ECAT HR PET Scanner. IEEE Trans. Nucl. Sci. 2013, 60, 797-801. [CrossRef]

25. Kidera, D.; Kihara, K.; Akamatsu, G.; Mikasa, S.; Taniguchi, T.; Tsutsui, Y.; Takeshita, T.; Maebatake, A.; Miwa, K.; Sasaki, M. The edge artifact in the point-spread function-based PET reconstruction at different sphere-to-background ratios of radioactivity. Ann. Nucl. Med. 2016, 30, 97-103. [CrossRef] [PubMed]

26. Mayer, A.T.; Natarajan, A.; Gordon, S.R.; Maute, R.L.; McCracken, M.N.; Ring, A.M.; Weissman, I.L.; Gambhir, S.S. Practical Immuno-PET Radiotracer Design Considerations for Human Immune Checkpoint Imaging. J. Nucl. Med. 2017, 58, 538-546. [CrossRef] [PubMed]

(C) 2019 by the authors. Licensee MDPI, Basel, Switzerland. This article is an open access article distributed under the terms and conditions of the Creative Commons Attribution (CC BY) license (http://creativecommons.org/licenses/by/4.0/). 